\title{
Evaluation of Performance of Kernel-Based Feature Extraction Techniques for Face Recognition System
}

\author{
${ }^{1}$ Fenwa Olusayo D., ${ }^{1}$ Ajala Funmilola A. and ${ }^{2}$ Makinde Bukola O., \\ ${ }^{1}$ Department of Computer Science and Engr. Ladoke Akintola University of Technology, Ogbomoso \\ Oyo State Nigeria \\ ${ }^{2}$ Department of Computer Science, Osun State College of Technology, Esa-Oke, Osun State Nigeria. \\ Corresponding Authors email: (odfenwa@lautech.edu.ng, faajala@lautech.edu.ng \\ shalombookey@yahoo.com)
}

\begin{abstract}
Face recognition is considered to be one of the most reliable biometrics where security issues are of concerned. Feature extraction which is a functional block of a face recognition system becomes a critical problem when there is need to obtain the best feature with minimum classification error and low running time. Most existing face recognition systems have adopted different non-linear feature extraction techniques for face recognition but identification of the most suitable non-linear kernel variants for these systems remain an open problem. Hence, this research work analyzed the performance of three kernel feature extraction technique (Kernel Principal Component Analysis, Kernel Linear Discriminant Analysis and Kernel Independent Component Analysis) for face recognition system. A database of 360 face images was created by obtaining facial images from LAUTECH Biometric Research Group consisting of six facial expressions of 60 persons. Images were preprocessed (gray scaling, cropping and histogram equalization) and the kernel variants were used to extract distinctive features and reduce the dimensionality of each of the images from 600x800 pixels to four smaller dimensions: 50x50, 100x100, 150x150 and 200x200 pixels. Euclidean Distance similarity measure was used for classification. The performance of the three kernel variants was evaluated for face recognition system using 180 images for training and 180 images for testing using Recognition Accuracy (RA) and Recognition Time (RT). Empirical results indicate that KLDA performs best for face recognition system with an average accuracy of $94.52 \%$. The larger image dimension also results in better recognition performance. We intend to experiment on other classifiers for face recognition system in our future work.
\end{abstract}

Keywords - Biometrics, Face, Feature extraction, Kernel, KICA, KPCA, KLDA, Linear, Non-linear

\section{INTRODUCTION}

The face is the primary focus of attention in social intercourse playing major role in conveying identity and emotion and if damaged, may be impossible to physically recognize people (Bansal and Pankaj, 2013). It is one of the biometric methods that acquire the qualities of not requiring cooperation of the test subject, beneficial to security and surveillance, does not require expensive equipment, totally non-alterable, does not carry any health risk, reliable and stable. Hence its details are phenotypically unique (Adedeji et al, 2012; Falohun et al, 2013).

Face recognition is the identification of a person from an image of their face. It is successful application of image analysis and pattern recognition from video or pictures using databases of face images (Rabia and Hamid, 2009). It is used for two primary tasks: identification (one-to-many matching) and verification (one-to-one matching) (Yakub et. al, 2017). It can be used in conjunction with other biometric methods such as iris, fingerprints and so on to enhance performance. There are predominant approaches to face recognition problem leading to development of different algorithms for face recognition systems which is 
a system that recognizes human face using characteristic or features. Feature extraction which is a functional block in face recognition system becomes a critical problem when there is need to obtain the best feature with minimum classification error and low running time. Most existing systems have adopted different linear and non-linear feature extraction techniques but identification of the most suitable variants remains an open problem.

This paper analyses the performance of three different non-linear kernel feature extraction techniques for face recognition system: Kernel Principal Component Analysis (KPCA), Kernel Independent Component Analysis (KICA) and Kernel Linear Discriminant Analysis (KLDA)

Section 2 and 3 discussed literature review and previous works related to our study while Section 4 gives detail of our research methodology. Experimental design and results was discussed in Section 5 while our conclusion/ contributions along with the future work are given in Section 6.

\section{LITERATURE REVIEW}

Face recognition is considered to be one of the most reliable biometric when security issues are taken into concern. For this, feature extraction becomes a critical problem when there is need to obtain the best discriminant feature. Different methods are used for extraction of facial feature which are classified broadly into linear and non-linear subspaces. Linear subspaces or methods perform a linear dimension reduction. Here, the face vectors/features are projected to the basis vectors, the projection coefficients are used as feature representation of each face images and approaches are Principal Component Analysis (PCA) family, Independent Component Analysis (ICA) family and Linear Discriminant Analysis (LDA) family. Recognition tasks could not be sufficiently fulfilled by linear methods because of their inadequacy to represent the complex and non-linear variations of real face images especially when face patterns are subjected to large variations due to head pose, illumination, aging and so on. (Ruiz and Lopez, 2001). Non-linear subspaces or methods are non-linear extension of linear methods. It combines the nonlinear kernel tricks with the linear subspaces providing better representations and lower error rates for face recognition. (Yang, 2002 and Kwang, Keechul and Hang, 2002). It calculates the higher order statistics of images unlike linear methods that consider only second order statistics and can discover the underlying structure of face images which normally resides on a non-linear manifold when there are variations in facial expression. Most common approaches are Kernel PCA, kernel ICA and Kernel LDA.

\subsection{Kernel Principal Component Analysis (KPCA)}

KPCA is a non-linear form of PCA which better exploits the complicated structure of high dimensional features; it allows generalization of standard PCA to non-linear dimensionality reduction (Scholkopf et al, 1999). Hence, it provides a replacement which takes into account higher order correlations. The basic idea is to map the input space into a feature space via non-linear mapping and then compute the principal components in that feature space. The mapping is made implicitly using kernel functions which encourages the data to become separable in feature space rather than reliant in the original input space.

\subsection{Kernel Independent Component Analysis (KICA)}

KICA combines the strength of the kernel and Independent Component Analysis (ICA) approach. First, images are mapped to high dimensional kernel space by using non-linear mapping, and then ICA is applied to extract the non-linear independent components in the face images. (Vankayalapati, 2008). It involves calculating the dot products of two vectors in 
high dimensional feature set with a kernel function, whitening operation followed by rotation operation using infomax iterative algorithm.

\subsection{Kernel Linear Discriminant Analysis (KLDA)}

The kernel idea for LDA was used to find non-linear directions by first mapping the data non-linearly into some feature space and computing the Linear discriminant there, thus implicitly yielding a non-linear discriminant in input space. (Huang et al, 2007).

\section{RELATE WORKS}

Adedeji et.al (2012) evaluated the performance of Optimized PCA (OPCA) and Projection Combined PCA $\left(\mathrm{PC}^{2} \mathrm{~A}\right)$ methods in black facial images using facial image recognition based on the following parameters: recognition accuracy, total training time and average recognition time. A database of 252 images with different facial expressions and lightening conditions were created. The result of evaluation using MATLAB environments between both algorithms showed that OPCA and $\mathrm{PC}^{2} \mathrm{~A}$ with $50 \times 50$ and $100 \times 100$ pixels gave recognition accuracy between $96 \%$ to $64 \%$ and between $95 \%$ to $60 \%$ respectively. Considering other parameters, overall results indicated that OPCA performed better than $\mathrm{PC}^{2} \mathrm{~A}$.

Bansal and Pankaj (2013) evaluated the performance of face recognition using PCA and Normalized-PCA(NPCA) and experiments are carried out on ORL, Indian Face database and Georgia Tech face database which contain variability in expression, pose and facial details. The results obtained for the two methods were compared by varying the number of training images and found out that as the number of training images increases, efficiency also increases. The result also showed that N-PCA gives better results than PCA.

Suganya and Menak (2014) carried out an evaluation performance of two important face recognition algorithms namely Principal Component Analysis (PCA) and Linear Discriminant Analysis (LDA). These algorithms are implemented in MATLAB considering recognition rate and verification rate and the performance is tested with ORL database. The results indicated that LDA outperforms PCA when training set is large whereas PCA outperforms LDA when training set is small. The recognition percentage is quite high with LDA compared to PCA for the same number of samples.

Face recognition and detection techniques was studied by Kaushik, Dubey and Abhimanyu (2014) and experiments was carried out using the algorithms like Principal Component Analysis (PCA), Kernel Principal Component Analysis (KPCA), Linear Discriminant Analysis (LDA) and Line Edge Map (LEM) in MATLAB environment on the basis of recognition accuracy and time used in face recognition procedure. Database containing 20 face images with two expressions of face were used for the experiments. The results showed that the best algorithm for face recognition is LEM technique after considering other metrics.

Aluko et.al, (2015) reported a performance evaluation of PCA-based techniques (PCA, BPCA and PCA-ANN) for face image recognition considering the quantitative effects of varying eigenvectors on recognition rate and time with respect to single image resolution. The experiments using MATLAB environment was performed on a created database consisting black facial images. The evaluation of the three PCA-based system indicated that PCA-ANN technique gave the best recognition rate with aa trade-off in recognition time.

Kamble et.al, (2015) studied Kernel Eigen faces Framework for Feature Extraction and Face Recognition using PCA, LDA and KPCA for performance evaluation. The methods were implemented for feature extraction and recognition on MATLAB environment and tested on 
two well-known face image databases ORL and JAFFE. The result showed that KPCA performed better than PCA and LDA on both databases with tradeoff between computation complexity and recognition accuracy. Performance evaluation of different support vector machine kernels for face emotion recognition considering radial basis function (RBF), linear function (LF), quadratic function $(\mathrm{QF})$ and polynomial function (PF) for classification of seven face images was carried out by Adeyanju, Omidiora and Oyedokun. The experiment was carried out using MATLAB and four different dimensions of images (50x50, 100x100, 150x150 and 200x200 pixels) were taking into consideration. The results showed that Quadratic Function Kernel outperformed the other three kernels in term of percentage accuracy.

\section{RESEARCH METHODOLOGY}

The basic stages involved in this work are: face acquisition/data splitting, image preprocessing, feature extraction, training and testing. Each step with respect to our work is discussed as follows:

\subsection{Face Acquisition/Data splitting}

This is the entry point of the face recognition process where the face under consideration is presented to the system. Face database used was obtained from LAUTECH Biometric Research Group. The obtained images were captured with a CMITECH Iris digital camera at different times, under different illumination, different facial expressions (open / closed eyes, smiling / not smiling) and converted into values suitable for processing by the computer. The camera resolution was $600 \times 800$ pixels. Some of the images from the database are presented in Figure 1. The images were divided into two sets: training and testing sets. The face database had a total of 360 face images, $50 \%$ were taken for training and placed into a folder called "TrainImage" while the remaining 50\% were taken for testing and placed into a folder called "TestImage".

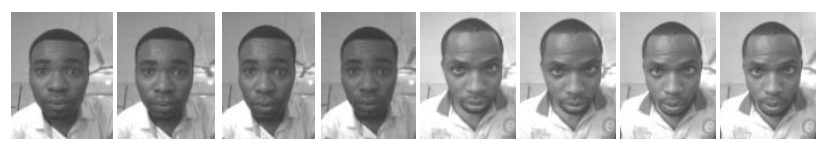

Figure 1. Raw images from the database

\subsection{Image preprocessing}

This stage involves face normalization which is used to compensate for position and illumination so that the variance can be minimize. The preprocessing steps considered were:

a. Grayscale: the coloured images were converted into grayscale which was two dimensional, so as to make the image suitable for processing. Some of the images already converted to grayscale were presented in Figure 2.

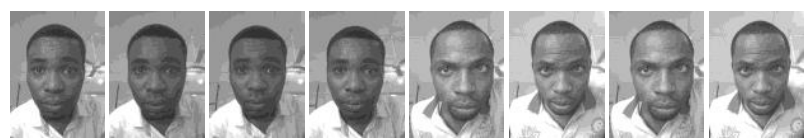

Figure 2. Gray Scaled Images

b. Cropping: face images were cropped out from their original captured images to required pixels $(50 \times 50,100 \times 100,150 \times 150$ and $200 \times 200)$ in order to extract major features like eyes, nose, eyelid and lips using image resize function in MATLAB and also to investigate the effect of varying dimension. Some of the cropped images were presented in Figure 3. 


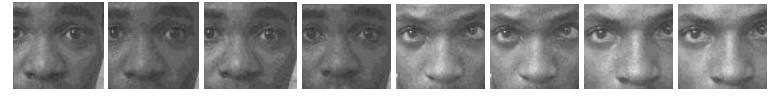

Figure 3. Cropped Image

c. Histogram Equalization: A histogram of the face was computed to compensate for lighting changes in the image. It improved the contrast in the images by stretching out the intensity range and enhances the brightness in the grayscale images for clearer recognition. Some of the equalized images were presented in Figure 4.

Figure 4. Equalized Images

\subsection{Feature Extraction}

The relevant features of the pre-processed face images were extracted using the selected three kernel variants (KPCA, KLDA and KICA) while the irrelevant features were discarded.

\subsection{Training:}

The images in the "TrainImage" database was train using each of the selected feature extraction technique.

\subsection{Testing/Recognition}

Comparison of the test images with those of the trained images was done at this stage to identify the class the tested images belongs. Testing/Recognition: The testing database was used to test the performance of the feature extraction techniques using Euclidean distance measure to classify and obtain the similarity level. Graphical User Interface of the designed face recognition system for the evaluation of the selected feature extraction techniques is shown in figure 5. 


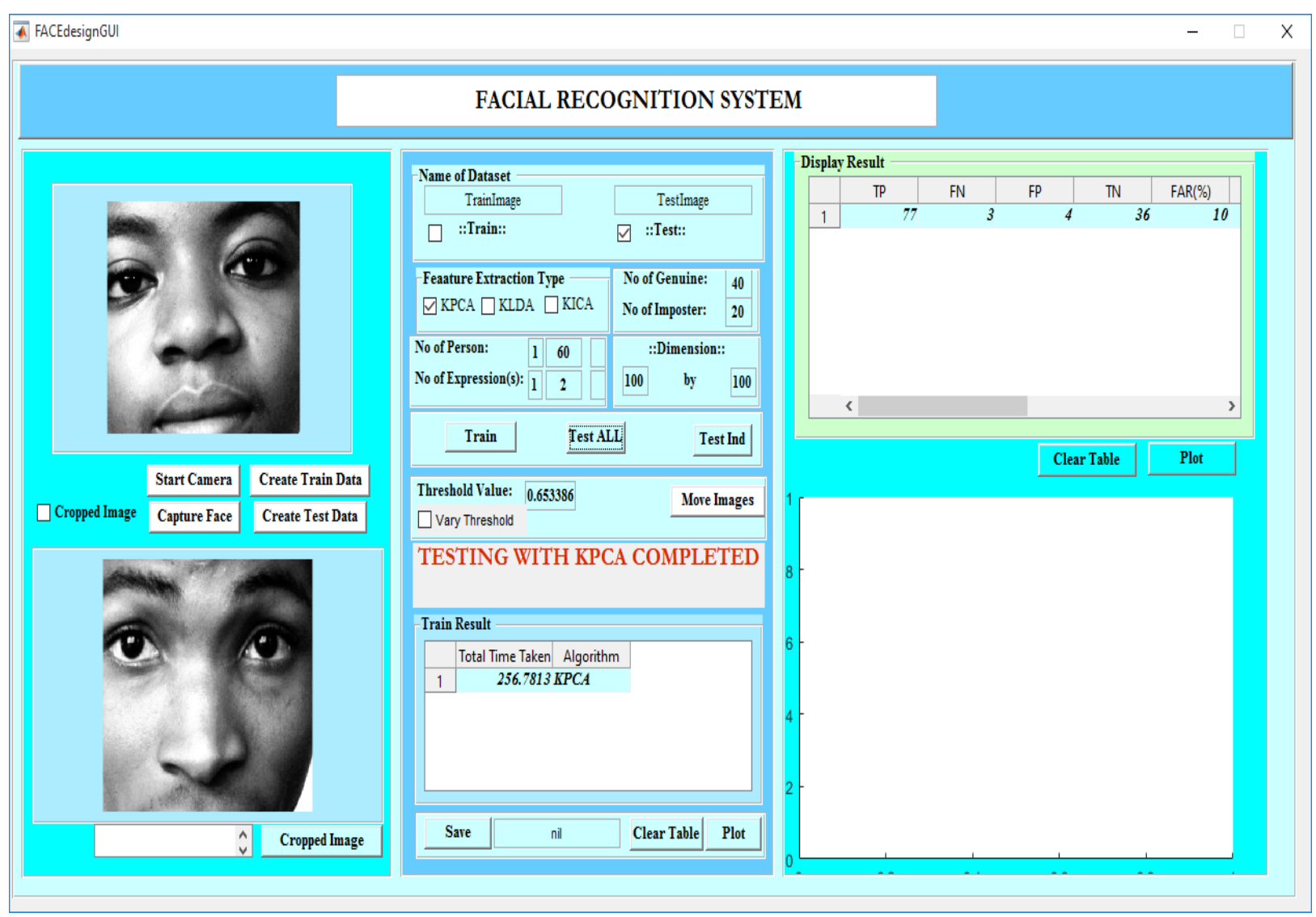

\section{EXPERIMENTAL RESULTS AND DISCUSSION}

The experiments were conducted using MATLAB programming environment with a total of 360 images using a percentage split (50\% for training and 50\% for testing) experimental setup. The training set contains a set of four (4) images per person from 45 subjects with varying side views (poses), face expressions and environmental conditions (lighting, rainy etc.). The testing set contains two (2) remaining images of those 45 subjects and the six (6) untrained images of the other fifteen (15) subjects with different side views (poses) or face expressions or environmental conditions. These represents 6 images per sixty (60) persons. The three kernel feature extraction techniques were tested on the four reduced image

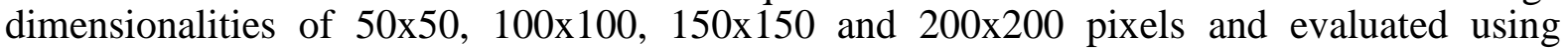
recognition accuracy and recognition time as performance metrics.

Experimental results for recognition accuracy and recognition time are shown in Table $1 \& 2$ respectively. Overall, the performances of the three kernel feature extraction techniques were high with the minimum average accuracy of $83.33 \%$ obtained by KICA kernel on the reduced image of 50X50. Nevertheless, KLDA outperformed other kernel variants (KPCA and KICA) with the highest average recognition accuracy of $96.95 \%$ when the 200 X200 reduced pixel images were used. In term of ranking the kernels based on descending order of their accuracy performance, the KPCA followed closely while KICA take the least.

The obtained results showed that the higher the dimension, the higher the accuracy. From these results, it is shown that KLDA attained the highest accuracy for all the dimension sizes followed by KPCA while KICA took the least. This implied that each of the kernel variants performed better as the dimension size increases thereby increasing the accuracy. This implied that the more the feature extracted, the higher the accuracy. The reason for that high 


\begin{tabular}{|c|c|c|c|}
\hline \multirow{2}{*}{$\begin{array}{c}\text { Image } \\
\text { Dimension }\end{array}$} & \multicolumn{3}{|c|}{ Kernel Variants } \\
\cline { 2 - 4 } & $\mathrm{KPCA}(\%)$ & $\mathrm{KLDA}(\%)$ & $\mathrm{KICA}(\%)$ \\
\hline $50 \times 50$ & 87.23 & 91.67 & 83.33 \\
\hline $100 \times 100$ & 89.45 & 93.89 & 86.11 \\
\hline $150 \times 150$ & 92.22 & 95.56 & 89.72 \\
\hline $200 \times 200$ & 93.61 & 96.95 & 92.22 \\
\hline
\end{tabular}

performance of KLDA is because of its discriminative power embedded in training stage by maximizing the between class scatter matrices and minimizing the within class scatter matrix. KLDA uses class information while KPCA and KICA do not consider any class information. They take all data as one entity.

Recognition time in this work is defined as the time spent by each of the kernel variants to test all the 180 images during experiments at varying dimension. This excludes time taken for preprocessing. KPCA was efficient taking less than 3 minutes for all dimension sizes followed closely was the KLDA. KICA took more than 3 minutes

for all the dimension sizes. The results showed that the higher the dimension sizes, the higher the time taken by each of the feature extraction techniques to implement because the pixel values increase as the dimension increases therefore more features are extracted thereby increasing the recognition time.

Overall, it is shown that KPCA has the shortest time frame for all dimension sizes because it does not require nonlinear optimization but only solution of an eigen value problem. Following KPCA closely is KLDA while KICA took the highest time because it involves an iterative process so it takes more time to converge.

Table 1: Recognition Accuracy Evaluation Results 
Table 2: Results Showing Recognition Time

\begin{tabular}{|c|c|c|c|}
\hline \multirow{2}{*}{$\begin{array}{c}\text { Image } \\
\text { Dimension }\end{array}$} & \multicolumn{3}{|c|}{ Kernel Variants } \\
\cline { 2 - 4 } & $\begin{array}{c}\text { KPCA(in } \\
\text { sec) }\end{array}$ & $\begin{array}{c}\text { KLDA(in } \\
\text { sec) }\end{array}$ & $\begin{array}{l}\text { KICA(in } \\
\text { sec) }\end{array}$ \\
\hline $50 \times 50$ & 2.89 & 2.93 & 2.29 \\
\hline $100 \times 100$ & 2.83 & 2.87 & 4.12 \\
\hline $150 \times 150$ & 2.83 & 2.86 & 6.07 \\
\hline $200 \times 200$ & 2.84 & 2.87 & 8.54 \\
\hline
\end{tabular}

Experiment showed that KLDA was able to extract the most discriminant features in the feature space which is equivalent to extracting the most discriminant nonlinear features in the original input space. KLDA uses class information and thereby separated the projected images quite well unlike others that smeared the classes. This provides an explanation to the good performance achieved by KLDA. KPCA is able to extract nonlinear features, allows utilization of different kernel functions and performed well in uncontrolled situations of varying illumination and slight change in expression and pose. The performance achieved by the KICA method indicates that face representation using independent basis images is not effective when the images contain pose, scale or lighting variation.

\section{CONCLUSION}

An evaluation of three different kernel-based feature extraction techniques for the classification of images has been presented in this paper. KPCA, KLDA and KICA techniques were evaluated to consider the quantitative effects of feature extraction methods on recognition accuracy and recognition time with respect to a varying pixel resolutions (50x50, 100x100, 150x150 and 200x200) and Euclidean distance measures as classifiers. The experiment was performed on black facial images under different face views, expression and illumination in an uncontrolled environment.

The performance evaluation showed that KLDA feature extraction technique gave the best recognition accuracy with a trade-off in recognition time with different pixel resolutions considered. The reason for that high performance of KLDA is because of its discriminative power embedded in training stage by maximizing the between class scatter matrices and minimizing the within class scatter matrix. Also, the recognition accuracy of KPCA and KICA increases with increasing pixel resolutions.

Our future work will investigate larger image database for evaluation, compare the performance of different distance measures (classifiers) and images will be taken under same facial conditions like environment and illumination. 


\section{REFERENCES}

Adedeji Oluyinka Titilayo, Omidiora Elijah Olusayo, Olabiyisi Stephen Olatunde and Adigun Abimbola Adebisi (2012): "Performance Evaluation of Optimized PCA and Projection Combined PCA methods in Facial Images". Journal of Computations and Modeling, 2(3), pp 17-29.

Adeyanju I.A, Omidiora E. O. and Oyedokun O.F.(2015): 'Performance Evaluation of Different Support Vector Machine Kernels for Face Emotion Recognition', Intelligent System Conference, November 10-11 2015, London, UK.978-1-4673-7606 IEEE, pp 804806.

Aluko J. Olubunmi, Omidiora E. Olusayo, Adetunji A. Bola and Odeniyi O. Ayodeji (2015): "Performance Evaluation of Selected Principal Component Analysis-Based Techniques for Face Image Recognition". International Journal of Scientific \&Technology Research, 4(1), pp $35-41$.

Bansal Ajay Kumar and Pankaj Chawla (2013); "Performance Evaluation of Face Recognition using PCA and N-PCA". International Journal of Computer Applications (09758887), 76(8), pp 14-20

Falohun A. S, Omidiora E.O, Fakolujo A.O, Ojo.J.A. (May 2013), "Development of an IrisBased Access Control System Using a Two-Level Segmentation Approach" International Journal of Computer Trends and Technology (IJCTT), 4(5) ISSN: 2231-2803 pp 1318-1326

Huang Jian, Pong C. Yuen, Wen-Sheng Chen, and Jian Huang Lai (2007): 'Choosing Parameters of Kernel Subspace LDA for Recognition of Face Images Under Pose and Illumination Variations', IEEE Transactions on systems, man, and cybernetics-Part B: Cybernetics, 37(4), August 2007.

Kamble Megha A. Sanjay L. Nalbalwar, Swarali P. Sheth (June 2015) "Kernel Eigenfaces Framework for Feature Extraction and Face Recognition", International Journal of Emerging Technology and Advanced Engineering (ISSN 2250-2459, ISO 9001:2008 Certified Journal, 5(6), pp 506-511

Kaushik Sangeeta, R. B. Dubey and Abhimanyu Madan, (2014) "Study of Face Recognition Techniques" International Journal of Advanced Computer Research 4(17) pp 939-949.

Kwang In Kim,Keechul Jung, Hang Joon Kim, (2002) "Face Recognition using kernel Principal Component Analysis", IEEE Journals \& Magazines Signal Processing Letters, 9, pp $40-42$.

Rabia Jafri and Hamid R. Arabnia (2009): “A survey of Face Recognition Techniques". Journal of Information Processing Systems, 5(2), pp 41-68

Ruiz, A., López de Teruel P.E.,(2001) : Nonlinear Kernel-Based Statistical Pattern Analysis. IEEE Transactions on Neural Networks,12(1), pp.16-32.

Scholkopf, Bernhard, Smola, Alexander, and Muller, Klaus-Robert. (1999) "Kernel principal component analysis in Advances Kernel Methods \{Support Vector Learning\}",Pp 327-352.

Suganya S. and D. Menaka, (2014): "Performance Evaluation of Face Recognition Algorithms", International Journal on Recent and Innovation Trends in Computing and Communication ISSN: 2321-8169, 2(1), pp 135-140. 
Vankayalapati Hima Deepthi,(2008), “ Evaluation of Wavelet Based Linear Subspace Techniques for Face Recognition", Institute for Smart System-Technology Transportation Informatics Klagenfurt. Pp 1-114

Yakub K. Saheed, Moshood A. Hambali, Ismaeel A. Adeniji and Akeem F. Kadri (2017): 'Fingerprint Based Approach for Examination Clearance in Higher Insttutions', FUOYE Journal of Engineering and Technology, 2(1), ISSN 2579-0625.

Yang, M.H (2002): ' Kernel Eigenfaces vs. Kernel Fisherfaces: Face Recognition using Kernel Methods. In Proceedings 5th International Conference Automatic Face Gesture Recognition, Washington, DC, May 2002, pp. 215-220. 